\title{
Book Review / News / Information
}

\section{Klaus Meyer, Direct Investment in Economies in Transtition, Cheltenham: Edward Elgar 1998}

This book is a typical $\mathrm{PhD}$ in business studies. It is divided into four parts starting with two introductory chapters, a second part containing the conventional literature review and some theoretical reflections, the third part as an emiprical analysis of foreign direct investment in transition economies, which covers nearly half of the volume and finally conclusions are presented. The theoretical part two is certainly not the strength of the book, but may serve as an introduction into the general research programme of the work. The connection between theory and the empirical analysis of part three remains very loose, what is problably not the fault of the author, but a result of the Britisch $\mathrm{PhD}$ culture, where a successful $\mathrm{PhD}$ thesis has to represent three publishable articles. However, for readers interested into determinants of FDI in CentralEast European econmies the advantage is, that they can just start to read on page 117. Here the inforamtive value of the study can be found. A survey is elaborated and explained in great detail. The findings are carefully analysed and one conclusion, that FDI is not a "kick-starter" of economic development is very convincing. The author himself seems to be disappointed by the theoretical fundamentals of his research and points towards the need of further research, the search for a "new theory of the firm" in particular. Those readers not willing to join in for this journey may simply take the empirical findings as useful orientation for the behaviour of the firm within an environment of great uncertainty.

\section{Jens Hölscher, Chemnitz University of Technology}

\section{Cracow University of Economics, Department of Philosophy.}

\section{CALL FOR PAPERS}

"Pictures of the contemporary economic person".

25-26 November 1999

We would like our conference to answer the question: how does the economic person form, who he becomes, as well as who he should be, in the present times.

This conference is organized as part of the 75-th aniversary of our University and we expect guests from EBEN to participate in it as well. We have invited twenty guests from Poland and abroad who have confirmed their participation. For the remaining guests we provide free catering and accomodation at their expense. The coference fee is $50 \mathrm{zl}$.

\section{Contact: \\ ejkusak@cyf-kr.edu.pl}

Dr Kazimierz Sosenko, Cracow University of Economics, fax: (4812)4120628, phone: (4812) 6167372, e-mail: ejkusak@cyf_kr.edu.p 
„Industriemanager als soziale Konstrukteure betrieblicher Organisationsund Arbeitsstrukturen. Eine vergleichende soziologische Untersuchung in Unternehmen Ostdeutschlands, Polens und der Tschechischen Republik“

Am 6. Mai 1999 fand in Chemnitz ein Workshop statt, in dessen Mittelpunkt die Präsentation und Diskussion von Ergebnissen eines Forschungsprojektes zu obigem Thema stand. Der Workshop wurde durch zwei weitere Beiträge abgerundet, die sich ebenfalls mit Problemen von Managern im Transformationsprozeß befaßten.

An dem vom Institut für Wirtschafts- und Sozialforschung Chemnitz e.V. (WISOC) organisierten und von der Volkswagen Stiftung geförderten Workshop nahmen insgesamt 19 WissenschaftlerInnen aus Deutschland, Polen und der Tschechischen Republik teil.

Ralph-Elmar Lungwitz (WISOC) gab zunächst einen Überblick über das Forschungsprojekt und ging insbesondere auf dessen theoretische Grundlagen sowie forschungsleitende Fragestellungen ein. In dem Projekt, das noch bis zum Sommer 1999 läuft, werden drei forschungsleitende Fragestellungen bearbeitet:

1. Was sind typische Pfade der Entwicklung betrieblicher Organisations- und Arbeitsstrukturen, nachdem die Grundlagen einer marktwirtschaftlichen Ordnung etabliert sind?

2. Worauf gründet sich das organisationsgenerierende Handeln von Eigentümern und Managern und zu welchen typischen Resultaten führt es?

3. In welchem Maße orientieren sich Manager und Eigentümer an „modernen“ Organisations- und Managementkonzepten, wie z.B. Gruppenarbeit, Kontinuierlicher Verbesserungsproze $\beta$, Gründung von Cost- oder Profitzentren?

Das Projekt, in dem Wissenschaftler der drei Untersuchungsländer zusammenarbeiten, beruht auf betrieblichen Fallstudien und folgt der Methode des Paarvergleiches. Es wurde ein Sample von sechs Betrieben pro Land, d.h. insgesamt von 18 Betrieben untersucht. Die Untersuchung wurde bewußt auf drei Branchen konzentriert, und zwar die Textil-und Bekleidungsindustrie, den Maschinenbau und die Automobilzuliferindustrie.

Nach einer kurzen Diskussion, die sich vor allem um den Begriff der „sozialen Konstruktion“ drehte, stellten weitere Mitglieder des Projektteams zunächst länderspezifische Befunde vor: Evelyn Preusche (WISOC) zu Ostdeutschland, Ivana Hollerova (Karlsuniversität Prag) zur Tschechischen Republik und Maria Gagacka/Anatol Peretiatkowicz (Politechnikum Radom) zu Polen. In Ergänzung hierzu referierte Stanislaw Rudolf (Universität Lodz) zu praktischen 
Erfahrungen aus polnischen Betrieben, die er in seiner Tätigkeit als Leiter des Centers of Participative Management sammeln konnte.

Ralph-Elmar Lungwitz unternahm den Versuch, erste allgemeine Tendenzen im Vergleich zwischen den drei Ländern herauszuarbeiten, was aufgrund des aktuellen Forschungsstandes allerdings nur in thesenhafter und vorläufiger Form möglich war. Es zeigte sich, daß die vorgefundenen Unterschiede hinsichtlich der Beschaffenheit von Organisations- und Arbeitsstrukturen zwischen den Branchen deutlich größer waren als zwischen den einbezogenen Ländern. Offenbar folgt das organisationsgenerierende Halndeln von Eigentümern und Managern einer branchenspezifischen Handlungslogik. Charakteristisch für die Arbeitsorganisation in den Nähbereichen der Bekleidungsindustrie war ein bewußtes Festhalten am klassischen Muster hoher Spezialisierung in Verbindung mit dem individuellen Akkord. Diese Organisationsweise wurde von den Managern/Eigentümern der drei Untersuchungsländer als konkurrenzlos hinsichtlich ihrer Effektivität eingeschätzt und stieß auch auf die ausdrückliche Zustimmung der Belegschaftsvertreter, die den individuellen Akkord als die gerechteste Lohnform betrachteten. Im Unterschied dazu fanden sich im Maschinenbau vor dem Hintergrund hoher externer Flexibilitätsanforderungen sowohl Formen institutionalisierter Funktionsintegration als auch eines problemorientierten informellen Zusammenwirkens auf der Basis eines hohen Personalisierungsgrades der sozialen Beziehungen in den Betrieben. In der Automobilzulieferindustrie ließen sich zwei Typen von Betrieben unterscheiden: Während einige Betriebe an den klassischen Organisationsform der Massenproduktion festhielten, wurde andere, die sich im Besitz internationaler Unternehmen befanden, von diesen einer grundlegenden Reorganisation auf der Basis des Lean-Konzeptes unterzogen.

Sofern ostdeutsche, polnische und tschechische Manager organisatorische Veränderungen initiierten, waren diese entweder durch externe Zwänge unvermeidlich, oder sie stellten eine unmittelbare Reaktion auf konkrete innerbetriebliche Probleme dar. Intendierte Veränderungen mußten einen eindeutigen Beitrag zur Lösung dieser Probleme erwarten lassen. Die Einführung sogenannter moderner Organisationskonzepte um ihrer selbst willen wird mehrheitlich abgelehnt.

Ramona Alt und Reinhart Lang (Technische Universität Chemnitz) stellten Ergebnisse verschiedener von ihnen seit 1990 durchgeführter empirischer Untersuchungen zur Entwicklung im Führungsverständnis von Managern in Ostdeutschland vor. Gleichzeitig übten sie Kritik an den überwiegend stichpunktbezogenen, oft vorurteilsbehafteten Studien $\mathrm{zu}$ den ostdeutschen Managern, die insbesondere zu Beginn der 90er Jahre entstanden. Im Ergebnis der eigenen Untersuchungen konstatierten R. Lang/R Alt eine relative Stabilität von tayloristisch-bürokratischen Organisations- und Führungsauffassungen und eine abnehmende Partizipationsorientierung bei den Managern in 
Ostdeutschland. Als Ursache der Stabilität sehen die Referenten neben der Stabilität in den Wertorientierungen und einer biographischen Kontinuität unter anderen auch die kleinbetriebliche Spezifik und die Qualifikationsstruktur der Mitarbeiter. In der Diskussion zu diesem Beitrag wurde darauf aufmerksam gemacht, daß die Erwartungen der Beschäftigten an die Führungskräfte Einfluß auf das Führungsverhalten der Manager haben. Die relativ konfliktfreien Beziehungen zwischen den Akteuren ostdeutscher Betriebe lassen vermuten, daß es eine große Übereinstimmung zwischen dem Führungsverständnis der Manager und den diesbezüglichen Erwartungen der Beschäftigten gibt.

Ulrich Brinkmann (Universität Trier) sprach in seinem Beitrag über Wirklichkeitskonstruktionen ostdeutscher betrieblicher Akteure am Beispiel von Normalisierung und Krankheitsmetaphorik. So habe es trotz eines Mangels an Strategien für die Unternehmensrestrukturierung in der ersten Phase des Transformationsprozesses mehr oder minder explizite Leitbilder gegeben, die als Handlungsanleitungen dienten. Autoren dieser Leitbilder seien die betrieblichen Akteure gewesen. Das Streben nach Stabilität und Normalität in Umbruchzeiten habe, wie U. Brinkmann am Beispiel Ostdeutschlands zeigt, zu diskursiven Anleihen bei „semantischen Komplexen“ geführt, die außerhalb der betrieblichen Welt liegen. Ein typisches Verfahren sei dabei die Naturalisierung betrieblicher Vorgänge entlang der Krankheitsmetaphorik gewesen (,ein gesunder Betrieb sein"). Die auf die Anfangsphase der Transformation begrenzte Dauer dieser Leitbilder nimmt ihnen nach Auffassung von U. Brinkmann deshalb nicht ihre Relevanz, weil in diese Phase die wichtigsten strategischen Entscheidungen für die Betriebe fielen.

Ralph-Elmar Lungwitz, Evelyn Preusche 


\section{The Journal for East European Management Studies}

\section{CALL FOR PAPERS}

Sonderheft zu: „Privatisierung und Transformation in Mittel- und Osteuropa“

Die Transformation und Restrukturierung der Wirtschaft in Mittel- und Osteuropa ist auf das Engste mit der massiven Privatisierung ehemals staatlich geleiteter Betriebe verbunden. Die Privatisierung bildet, insbesondere für die wirtschaftliche Transformation, das Hauptinstrument zur Etablierung eines neuen Wirtschaftssystems. Das Sonderheft will diesen Aspekt aus mehreren Perspektiven betrachten. So sind neben Beiträgen aus der Organisations- und Managementforschung auch Vertreter der Volkswirtschaft aufgefordert, Artikel einzureichen.

Die eingesandten Beiträge können die folgenden Felder fokussieren:

- Konzepte, Ansätze und Pfade der Privatisierung von Unternehmen im Prozeß der Transformation

- Einfluß von Interessengruppen in privatisierten Unternehmen

- Institutioneller Wandel als Koordinations- und Managementproblem

- Rahmenbedingungen der Privatisierung

Der Schlußtermin für die Einreichung von Beiträgen ist der 20. Dezember 1999. Die Autoren werden bis zum 30. April 2000 über die Annahme/Ablehnung ihres Artikels benachrichtigt. Für Fragen wenden Sie sich bitte an die untenstehende Adresse.

\section{Kontakt:}

Ingo Winkler, Technische Universität Chemnitz, Lehrstuhl BWL V Reichenhainer Str. 39, 09107 Chemnitz, BRD

Tel.: $+49 / 371 / 531-4159$

Fax: $+49 /(0) 371 / 531-3987$

Email: ingo.winkler@wirtschaft.tu-chemnitz.de

Internet: http://www.tu-chemnitz.de/wirtschaft/bwl5/ 
The Journal for East European Management Studies

\section{CALL FOR PAPERS}

Special Issue on: Privatisation and Transformation in Middle and Eastern Europe

Economic transformation and restructuring in Central and Eastern Europe is closely linked to the massive privatisation of formerly state owned enterprises. In the economic transition privatisation is the main instrument to establish a new economic system. This special issue will address this aspect from different perspectives. Therefore besides contributions from the research on organisations and management also economists are called to submit papers.

The papers submitted can address the following points:

- Concepts, approaches and paths of privatisation of enterprises in transition,

- Influence of stakeholders within privatised enterprises,

- Institutional Change as problem of co-ordination and management and

- Conditions of privatisation.

The articles submitted should reach us until the 20 December 1999, al the latest. All authors will be notified about the status of acceptance until 30 April 2000. For further questions please use the address below.

\section{Contact:}

Ingo Winkler, Chemnitz University of Technology, Chair BWL V

Reichenhainer Str. 39, 09107 Chemnitz, Germany

$$
\begin{gathered}
\text { tel.: }+49 / 371 / 531-4159 \\
\text { fax: }+49 /(0) 371 / 531-3987
\end{gathered}
$$

Email: ingo.winkler@wirtschaft.tu-chemnitz.de

Internet: http://www.tu-chemnitz.de/wirtschaft/bw15/ 